\title{
Long-term results and risk factors influencing outcome of gas tamponade for myopic foveoschisis with foveal detachment
}

\author{
I-Hsin $\mathrm{Ma}^{1} \cdot$ Yi-Ting Hsieh ${ }^{1}$ Po-Ting Yeh ${ }^{1} \cdot$ Chang-Hao Yang ${ }^{1,2} \cdot$ Chung-May Yang ${ }^{1,2}$
}

Received: 3 February 2019 / Revised: 7 May 2019 / Accepted: 5 July 2019 / Published online: 12 August 2019

(c) The Author(s), under exclusive licence to The Royal College of Ophthalmologists 2019

\begin{abstract}
Objective To evaluate the long-term results of gas tamponade without vitrectomy in patients with myopic foveoschisis (FS) and foveal detachment (FD).

Methods Twenty eyes of 20 patients with myopic foveoschisis and foveal detachment were analysed retrospectively. All patients received an intravitreal injection of $0.2-0.3 \mathrm{~mL} \mathrm{C3F8}$ as an initial treatment. The patients were followed up for at least 18 months after the procedure. The refractive status, best-corrected visual acuity (BCVA), and anatomical abnormalities in macular area before and after the treatment were assessed and recorded.

Results After initial gas tamponade, FD resolved completely (defined as responders) in 14 eyes, resolved partially in 2 eyes (defined as partial responders), and worsened in 4 eyes (non-responders) at the 3-month follow-up visit. Vitrectomy was performed in four eyes that showed increased detachment (two cases) or developed macular hole (two cases). Repeated gas injection was performed in two recurrent cases after initial success at the 3-month follow-up, and the foveal detachment once again resolved. Overall, $70 \%$ of our cases demonstrated resolution of FD at the 3-month follow-up and the long-term responders to gas injection was $65 \%$. The risk factors for treatment failure were: (1) The presence of optical coherence tomography (OCT)-evident premacular membranes $(p=0.03)$ and (2) the height of FS measured $>588 \mu \mathrm{m}(p=0.009)$.

Conclusion Our data supported that gas tamponade may be effective as an alternative treatment for selected cases of myopic FS with FD. The procedure may be especially beneficial in patients without OCT-evident premacular membranes and have low height of FS.
\end{abstract}

\section{Introduction}

Pathological myopia, estimated to affect $0.9-3.1 \%$ of the world population, is defined as "high myopia with any posterior myopia-specific pathology from excessive axial elongation" and may lead to the development of sightthreatening eye diseases [1-3]. Structural changes in

Supplementary information The online version of this article (https:// doi.org/10.1038/s41433-019-0555-3) contains supplementary material, which is available to authorized users.

$\triangle$ Chung-May Yang

chungmay@ntu.edu.tw

1 Department of Ophthalmology, National Taiwan University Hospital, No. 7, Chung-Shan South Road, Zhongzheng District, Taipei, Taiwan

2 College of Medicine, National Taiwan University, No. 1, Section 1, Ren-Ai Road, Zhongzheng District, Taipei, Taiwan pathological myopia include thinning of the scleral and expansion of the globe, and, as a consequence, the development of posterior staphyloma and optic disc myopic conus [4-8]. Other changes within the retinal layers are increasingly noticed as optical coherence tomography (OCT) becomes more widely used, such as foveoschisis (FS), peripapillary detachment, tractional internal limiting membrane (ILM) detachment, and lamellar or full-thickness macular hole (LMH and FTMH, respectively), and these changes were filed under the category of myopic tractional maculopathy (MTM). The pathogenesis of myopic FS was proposed to be the result of dynamic forces between outward stretch of the posterior staphyloma and contralateral vitreoretinal traction [9]. In cases of myopic FS, 34.5-72\% of the cases may progress to develop foveal detachment (FD) [10-12]. FD marks poor prognosis of vision outcome in the eyes of pathologic myopia [10].

Vitrectomy with or without pealing of the ILM has been advocated for treating myopic FS [13-16]. However, controversies remain as the natural course of myopic FS may be 
Fig. 1 Examples of optical coherence tomographic (OCT) images showing the measurement of various parameters and vitreomacular interface abnormalities. a Central foveal thickness, the height of foveal detachment, and the height of foveoschisis are shown by the vertical lines. CFT central foveal thickness, FD foveal detachment, FS foveoschisis. b-1 premacular membranes (hollow arrows), b-2 peripapillary traction (arrow), and $\mathbf{b}-3$ nerve fibre layer schisis (asterisks) are shown

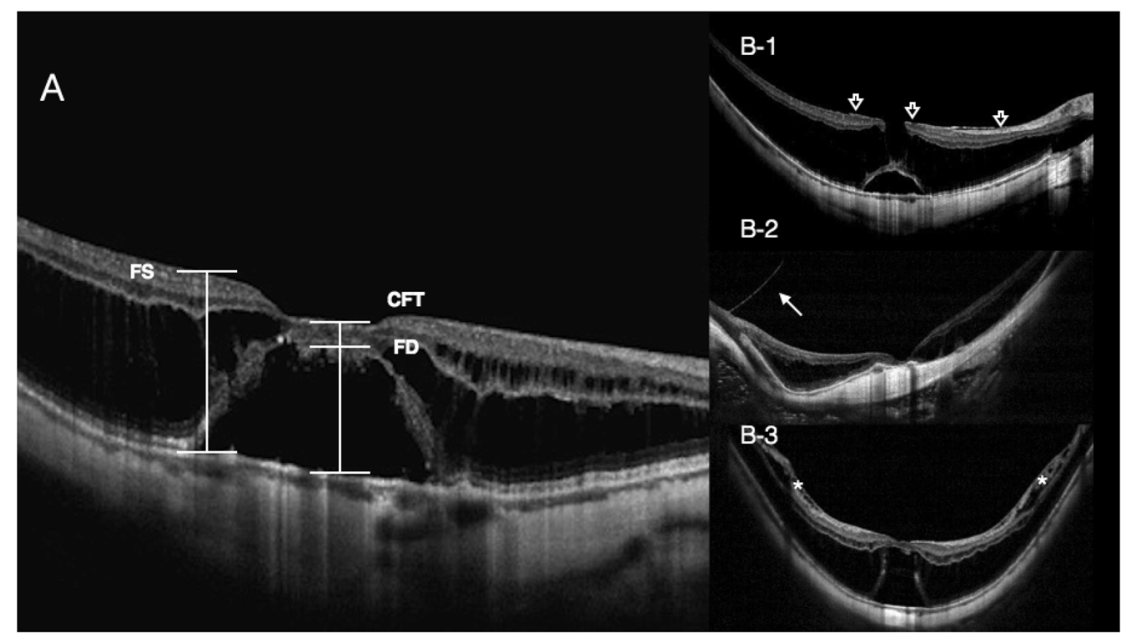

variable. Shimada et al. reported 207 patients with myopic FS, in which most showed stable clinical courses, and only $11.6 \%$ of them progressed during a follow-up period of $>2$ years [17]. In some cases, myopic FS may even improve with time [18]. On the other hand, the presence of FD and premacular membrane makes the formation of $\mathrm{MH}$ more likely. In addition, vitrectomy with ILM peeling, although highly effective to release traction, may have surgical risks, even if fovea-sparing technique was used [19]. Thus the optimal treatment option for myopic FS is still debatable.

In our previous study, we reported the feasibility of treating FD with gas tamponade alone in MTM patients [20]. The anatomical improvement rate was around 70\%; however, the study only had a small case number, and some patients were only followed for short periods. MTM complicated with FD and FS may fluctuate after gas injection, where some patients who responded well initially may relapse over time, and vice versa. Thus long-term follow-up with more cases is necessary to fully elucidate the effect of the gas treatment and to analyse the risk factors for disease progression [20]. Our hypothesis was that, after the injection, if the forces between gas expansion, out-pouching of the posterior staphyloma, and the traction of premacular membranes did not compromise the retinal structures, a new balance may be reached, enabling subfoveal fluid reabsorption or even improvement of retinoschisis. In this report, we examined 20 patients and monitored their longterm results to assess the effect of gas tamponade in treating MTM with FD and to identify possible risk factors that may predict poor outcome.

\section{Materials and methods}

Between November 2007 and August 2017, consecutive patients who have received gas tamponade for myopic FS with FD at National Taiwan University Hospital were reviewed. Patients were selected to have gas injection for FS and FD if the height of foveal elevation was $<1250 \mu \mathrm{m}$ (about 5 times of an average macular thickness). Informed consent was obtained from the patients after a thorough discussion of the pros and cons of gas injection and vitrectomy. Those fulfilling the following inclusion criteria were recruited in this retrospective study: (1) age $>18$ years; (2) high myopia with refraction $<-6.0 \mathrm{D}$ (spherical equivalent) or axial length $>26 \mathrm{~mm}$; (3) presence of inner and/or outer retinal schisis in the posterior pole combined with localized retinal detachment within the temporal arcades; (4) a minimum follow-up period of 18 months after the first gas tamponade; (5) if both eyes underwent the same treatment, only the first eye is included. Patients with $\mathrm{MH}$ or previously treated retinal detachment were excluded from the study, and those with concomitant peripheral degenerations or breaks that warrants treatment were also excluded. Informed consent was acquired upon first clinic visit for clinical research data acquisition. The study was approved by the Ethics Committee and Institutional Review Board of National Taiwan University Hospital and adhered to the Declaration of Helsinki.

Patients' preoperative data, including demographics, refractive error, lens status, axial length, and best-corrected visual acuity (BCVA) were retrieved and recorded. Preoperative OCT was analysed for the recording of central foveal thickness (CFT), the height of FD and FS, and vitreoretinal interface abnormalities. The height of FD was defined as the largest distance measured manually between the inner border of the retinal pigment epithelium (RPE) and the outer border of the neural retina at the foveal area. The CFT was measured at the same site as for FD, but the distance was from the RPE to the inner retinal surface. The height of FS was defined as the largest difference between the inner and outer borders of the neural retina within the area of FD (Fig. 1a). Vitreoretinal interface abnormalities were deemed as present if OCT showed one or more of the 


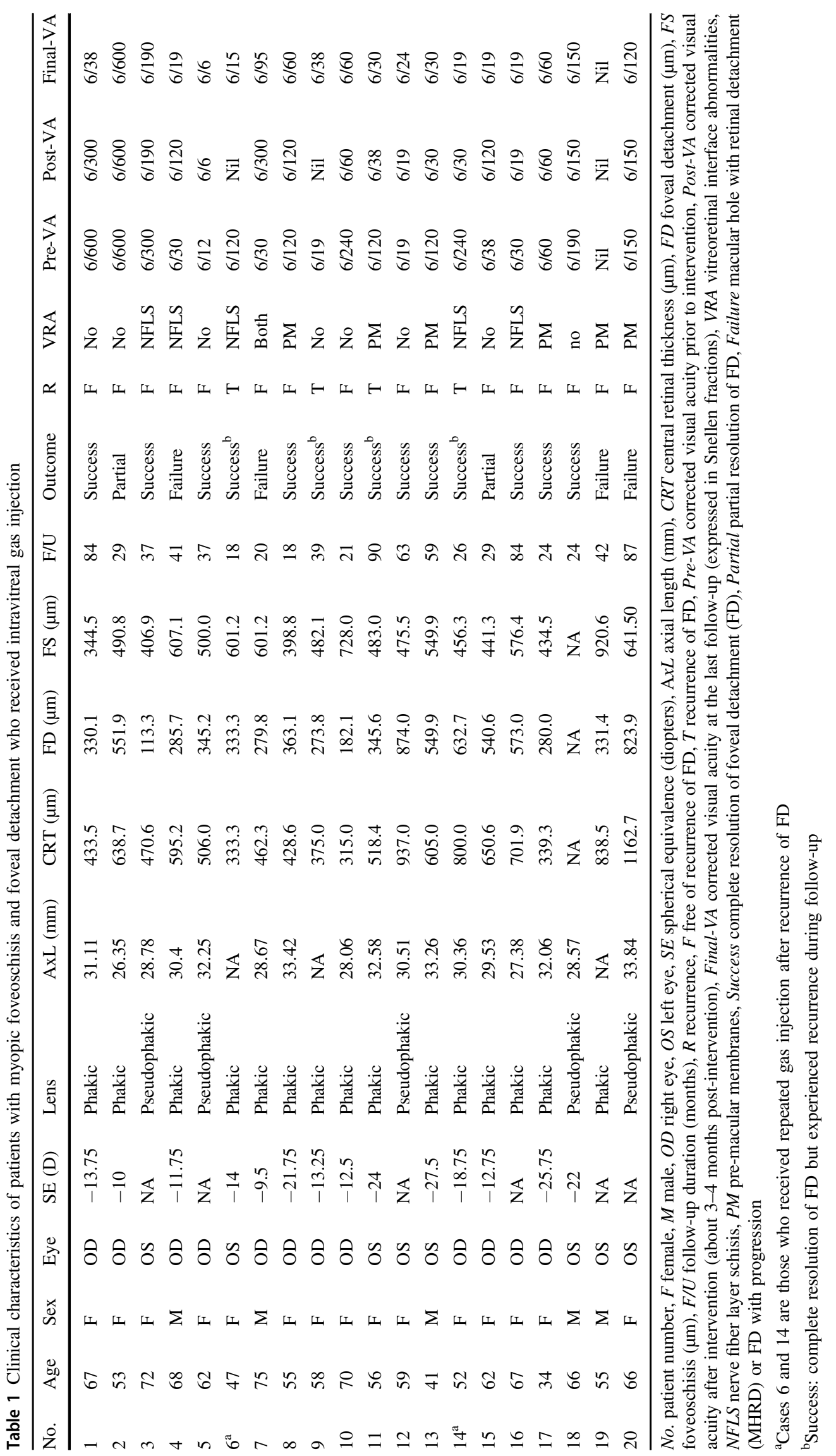


Fig. 2 Diagram illustrating the treatment courses and anatomical results of all the treated eyes. $F D$ foveal detachment, $F S$ foveoschisis, MTM myopic tractional maculopathy. Fragmented rectangle marks the treatment received. Gas intravitreal gas injection, $P P V$ vitrectomy with internal limiting membrane peeling. Numbers in brackets represents the patient count. *Resolution of FD, with or without FS. 'Partial responders referred to those with partial resolution of FD after gas injection only. ${ }^{\ddagger}$ Non-responders referred to those with either progression of FD or those complicated with macular hole. **Responders referred to those with complete resolution of FD with only gas injection

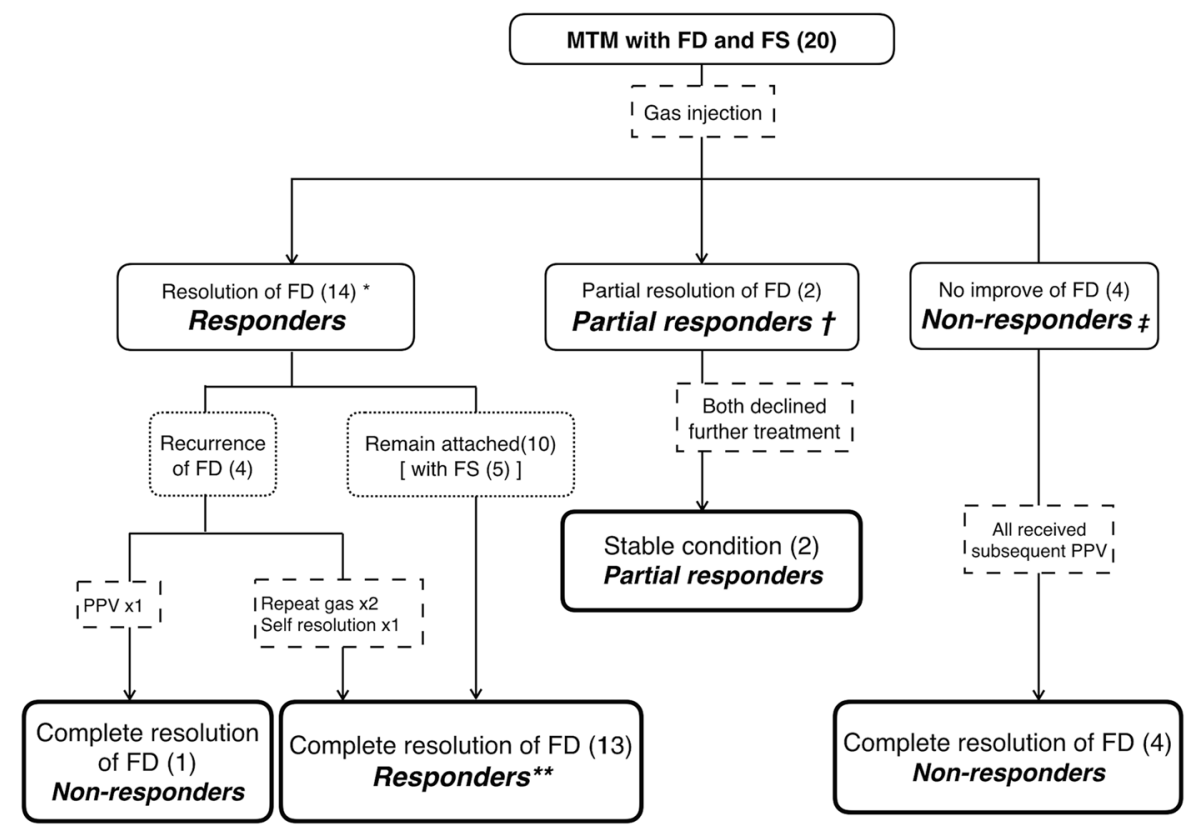

following three conditions: premacular membrane (Fig. 1b1), peripapillary traction (Fig. 1b-2), and schisis in the nerve fibre layer (Fig. 1b-3). Premacular membrane is defined as the presence of either epiretinal membrane (ERM) or vitreomacular traction (VMT). The reason that cases with OCT-identifiable ERM in our series were not excluded is that, in a high myopic eye, the absence of ERM on OCT does not preclude the actual presence of ERM, which we occasionally encounter during operation but not visible on preoperative OCT [21, 22]. Post-procedural recordings included BCVA and OCT analysis, focusing on the height of FD and FS, the evolution of vitreoretinal interface abnormalities, and the formation of $\mathrm{LMH}$ or FTMH.

Fundus photography and standard 6-, 9-, 10-mm OCT images of the macula were obtained for all participants using Cirrus ${ }^{\mathrm{TM}}$ HD spectral domain OCT (Carl Zeiss Meditec, Inc., Dublin, CA, USA) or Optovue Avanti RTVue XR OCT (Optovue, Inc., Fremont, CA, USA).

After proper disinfection and anterior chamber paracentesis, intravitreal injection of $0.2-0.3 \mathrm{~mL}$ perfluoropropane (C3F8) was performed using a 27-gauge needle through the temporal upper or lower pars plana at 3.75- and 3.25-mm posterior to the limbus in phakic and pseudophakic patients, respectively. Postoperatively, patients were advised to maintain a facedown position for 5 days and then take up a regular follow-up schedule.

We stratified the outcome at 3 months and at the last follow-up time into the following categories: complete resolution of FD with or without FS, partial resolution of FD and FS, worsening of FD, and complication of $\mathrm{MH}$.
Among which complete resolution of FD after primary injection, regardless of the status of FS, were defined as responders. Those with worsening of FD and complicated with $\mathrm{MH}$ were defined as non-responders; others were classified as partial responders.

For patients with limited improvement of FD within 3 months of gas injection and without other complications from the procedure, repeated gas tamponade to facilitate subretinal fluid absorption was proposed and performed with the patients' consent. An additional gas injection was also offered to patients with initial good response but subsequent recurrence of FD during follow-up. Recurrence was defined as re-emergence of FD at least 3 months after the primary documented outcome. Vitrectomy was carried out in patients with $\mathrm{MH}$ formation or as requested by the patient.

\section{Statistical analysis}

Age, refractive error, axial length, heights of FD, and CFT were compared between different outcomes using Student's $t$ test, assuming the similar variances. FS was analysed with Wilcoxon rank-sum test. Lens status and presence of premacular membranes were compared using Fisher's exact test. Statistical significance was considered as $p<0.05$. Optimal cut-off for statistically significant continuous variable was calculated by the receiver operating characteristics (ROC) curve analysis with assessment of the area under the curve (AUC) [23]. An AUC value of $>0.8$ was considered excellent, and the specificity and sensitivity of the cut-off point were also calculated. 


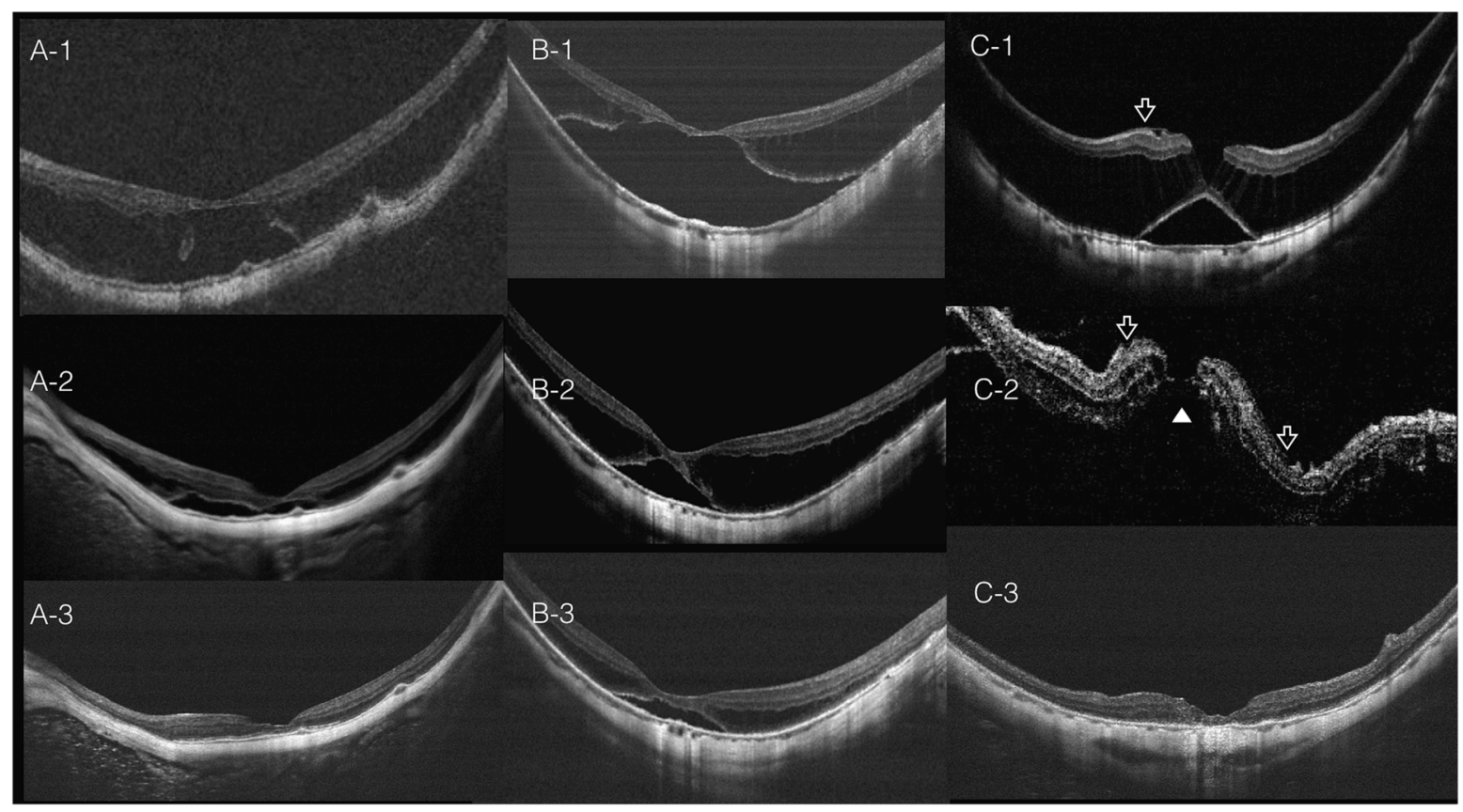

Fig. 3 a Representative optical coherence tomographic (OCT) images before and after intravitreal injection of $\mathrm{C} 3 \mathrm{~F} 8$ showing complete resolution of foveal detachment (FD) and foveoschisis (FS). a-1 FD and FS (measured $549.9 \mu \mathrm{m}$ ) were detected in preoperative OCT images. a-2 After gas injection, FD gradually decreased over several months. a-3 Reattachment and resolution of FS was achieved and sustained for 5 years. b OCT images before and after C3F8 intravitreal injections showing partial resolution of FD and FS. b-1 Preoperatively, FD and FS were noticed. b-2 Four weeks after gas injection, FD

\section{Results}

\section{Clinical characteristics}

Twenty eyes of twenty patients were enrolled in the study. Demographics are shown in Table 1. The average age was 59 years, ranging from 34 to 75 years; there were 5 men and 15 women. The mean follow-up time was 43.6 months, ranging from 18 to 90 months. The mean refractive error, documented as spherical equivalent, was $-16.95 \pm 5.92 \mathrm{D}$, ranging from -9.5 to $-27.5 \mathrm{D}$; the mean axial lengths was $30.42 \pm 2.19 \mathrm{~mm}$, ranging from 26.35 to $33.84 \mathrm{~mm}$. Five of the twenty eyes were pseudophakic upon initial presentation, others were phakic during intervention.

\section{Preoperative OCT findings}

Preoperative OCT revealed the average height of FD, height of FS, and central retina thickness were 410.2 $\pm 209.9 \mu \mathrm{m}$ $(113-874 \mu \mathrm{m}), 519.0 \pm 97.2 \mu \mathrm{m}(344-728 \mu \mathrm{m})$, and $565.8 \pm$ $212.4 \mu \mathrm{m}(315-1162 \mu \mathrm{m})$, respectively. Nine of the 20 patients had premacular membranes upon the diagnosis of FS and FD. significantly decreased, but FD persisted. b-3 Long-term follow-up showed persistent detachment but with less height than initial. c OCT images before and after C3F8 intravitreal injection of a non-responder who developed macular hole. c-1 FD, FS, and premacular membrane (hollow arrow) were noticed preoperatively. c-2 Three weeks after gas injection, retinal detachment associated with a full thickness macular hole (FTMH) was seen (arrow head) along with premacular membranes (hollow arrows). c-3 After vitrectomy, the retina was reattached with closure of the macular hole

\section{Outcomes}

We defined those who showed complete resolution of FD after a single or repeated gas injection as responders. After the initial gas injection, 14 out of 20 eyes (70\%) achieved complete resolution of $\mathrm{FD}$ at 3 months postinjection (responders); 2 patients had partial resolution of FD (partial responders); and four eyes had worsening of the disease (non-responders) (Fig. 2). Among the 14 responders, 5 eyes (36\%) also had complete resolution of FS (Fig. 3a), while the remaining 9 eyes $(64 \%)$ had partial resolution of FS (Fig. 3b). Of the 4 patients (20\%) who had progression of disease after initial treatment, 2 experienced progression of FD, and the other 2 developed $\mathrm{MH}$ with detachment (Fig. 3c). The two with progressive FD was documented on the first postinjection office visit and subsequent visit did not show improvement. The development of $\mathrm{MH}$ occurred at 3 weeks and 2 months postinjection separately.

Of the 14 initial responders, 4 experienced recurrence of FD. In these four eyes, two were treated successfully with an additional injection of $\mathrm{C} 3 \mathrm{~F} 8$, one resolved spontaneously over a period of several months, and one received vitrectomy on patient's request, which we included in the nonresponders group. 
Overall, the final responders were 13 eyes $(65 \%)$ with gas injection or with repeated gas injection. Partial responders were two eyes and showed no detectable changes in the detachment status during follow-up without additional injections. Non-responders were five eyes, including the four initial non-responders and one initial responder with FD recurrence, all achieving lasting FD resolutions without residual fluid or schisis after vitrectomy. Functionally, at the last follow-up, BCVA improved in 14 eyes, remained the same in 2 eyes, and worsened in 3 eyes. The functional outcome correlates closely with the anatomical outcome (Table 1.).

We compared the preoperative characteristics of the gas responders, partial responders, and non-responders to identify potential risk factors for poor prognosis after gas injection to provide guidance for future treatment. Statistical analysis comparing non-responders to the others showed that the presence of premacular membrane and the height of FS served as two significant risk factors for poor treatment response ( $p=0.03$, Fisher's exact test; and 0.009, Wilcoxon rank-sum test, respectively) (Supplement Table 1). With ROC depicting FS and the outcome of gas injection, we found that the AUC was 0.925 , and the optimal threshold of $588.8 \mu \mathrm{m}$ yielded a sensitivity of 0.87 and specificity of 1.0 for treatment failure (Fig. 4). We performed subgroup analysis by stratifying patients into groups with and without premacular membranes, and the result confirmed the negative impact of FS height in intravitreal gas injection independent of premacular membranes $(p<0.05$, Wilcoxon rank-sum test). The axial length between the two groups, however, showed no statistically significant difference. In the two patients who developed FTMH after the first injection of $\mathrm{C} 3 \mathrm{~F} 8$, the preoperative heights of FS were both $>588 \mu \mathrm{m}$, and both had evident premacular membranes on OCT sections preoperatively.

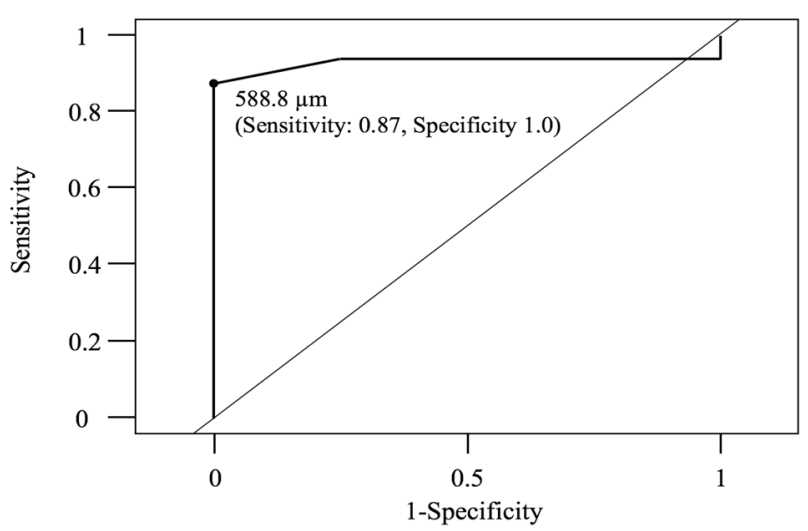

Fig. 4 Receiver operative curve depicting the height of foveoschisis and the outcome after gas injection. The area under curve was 0.925 , and a cut-off point at $588.8 \mu \mathrm{m}$ has a sensitivity of 0.87 and specificity of 1.0 for treatment failure

\section{Discussion}

In this study, we defined responders as those with resolution of FD after gas injection with or without resolution of FS. The significance of FD resolution in MTM is that it may improve functional outcome and, more importantly, decrease the risk of $\mathrm{MH}$ development. We selected the data at 3-month period and the last follow-up to investigate the difference between the initial response and the long-term results in order to better understand the exact effect of the gas treatment. Consistent with our previous study with fewer cases and a shorter follow-up period [20], the rate of foveal reattachment after one session of intravitreal gas injection was $70 \%$ at the 3-month period in this larger cohort. The favourable response was detected soon after gas injection; therefore, the improvement was mainly contributed by the gas treatment instead of the spontaneous resolution sometimes seen in such cases. However, during subsequent follow-up, some of the cases experienced recurrence. Because of the initial response to gas injection, repeated injection was offered to those cases with recurrent detachment, and the majority again obtained foveal reattachment. In the long-term follow-up, our results showed that 13 cases $(65 \%)$ attained complete resolution of FD with gas injections only and were deemed as responders. With resolution of FD, none developed $\mathrm{MH}$ during the follow-up period, and visual acuity also improved in these patients.

Important new findings were recognized in this study: (1) The presence of premacular membranes and the height of FS upon presentation were significant risk factors for potential treatment failure. (2) We identified that $\mathrm{MH}$ with retinal detachment, although rare, emerged as a complication of gas injection. (3) Repeated gas injections posted no specific risk in patients who did not have adverse response to the initial injection.

The discovery that the premacular membranes and the higher FS heights predict treatment failure is in concordance with Gaucher's description that MTM with FD and ERM was found to develop MH [10]. As the height of FS is measurable, quantitative analysis can be made. In our study, we found significant differences between responders and non-responders, with a mean FS height of 491.3 and $622.8 \mu \mathrm{m}$, respectively. Moreover, using ROC, we deducted a critical point of FS height at $588.8 \mu \mathrm{m}$, which had a high sensitivity and specificity to separate the favourable from the unfavourable outcome with gas tamponade. We believe that this could serve as a practical marker for eligibility screen prior to intravitreal gas injection. We proposed that the interaction between the expansion of intraocular gas and the retina was the major factor determining the final outcome. The stronger the traction of the premacular membranes, the less likely the injected gas may exert a beneficial effect on the retinal configuration. Although not all 
preretinal membranes can be seen on OCT images, our study suggests that only the eyes with OCT detectable membranes were prone to treatment failure. This result may indicate that either the traction is stronger if the membrane could be seen on OCT or gas induces more prominent traction on membranes visible by OCT, which, when severe, may result in treatment failure. In this case, vitrectomy is more suitable. Also, from our study, no treatment failure has been noticed after vitrectomy.

In our series, we encountered two cases of MH formation after gas injection. Although the development of MH may be the natural course of the disease, the close temporal association between gas tamponade and $\mathrm{MH}$ formation strongly suggests a cause-effect relationship. The characteristic features of these two cases include OCTidentifiable premacular membranes and higher height of FS than $588.8 \mu \mathrm{m}$.

In this study, we found that repeated gas injection had no major adverse impact on the macular condition such as decreased BCVA, increased FD, or FTMH formation. We observed that if the response to the first injection was favourable (i.e. total or partial resolution of FD), favourable responses could be expected for additional injections for those who had persistent partial FD or recurrent FD, regardless of VM interface abnormalities. We propose that, after the first injection, if the forces between gas expansion, out-pouching of the posterior staphyloma, and the traction of premacular membranes did not compromise the retinal structures, a new balance may be reached, enabling the retina layers to settle down gradually.

Our study was limited by its retrospective nature and the lack of controls. In addition, the case number was only modest, and only those patients with less severe FS were offered to have gas treatment (see "Materials and Methods" section); thus the success rate could be biased. Nevertheless, our long-term study did seem to support that gas tamponade can be used as an alternative treatment for selected cases of myopic FS with FD.

In conclusion, our results showed that preoperative OCT examinations may serve as an objective tool for selecting patients who may benefit from gas injections. Although the concept that tractional membranes may lead to poor prognosis was not new, it was not statistically demonstrated before. Further, in this study, we found a cut-off point of FS height of $<588 \mu \mathrm{m}$ and an absence of vitreoretinal traction membrane by OCT as two conditions for a favourable outcome after gas injection; also, those initial responders may have good response after repeated gas injection when recurrence of FD developed. Therefore, we suggest gas injection as a reasonable option for MTM cases with low FS height and no OCT-evident premacular membranes. Further study with more cases may be required to confirm our observations.

\section{Summary}

\section{What was known before}

- In myopic foveoschisis, many progress into foveal detachment, which marks poor prognosis of vision outcome.

- The evolution course of myopic traction maculopathy may be variable.

- Although vitrectomy with or without internal limiting membrane peeling has been widely used, the best treatment option for foveoschisis with foveal detachment remains controversial.

\section{What this study adds}

- Intravitreal gas injection could be an effective alternative to vitrectomy in selected cases of myopic traction maculopathy with foveoschisis and foveal detachment.

- The height of foveoschisis and the presence of premacular membrane were major factors related to the anatomical results after intravitreal gas injection.

- Repeated gas injection posts little additional risks for those who showed no complications after initial gas treatment.

Acknowledgements English Editing was provided by the Department of Medical Research at National Taiwan University Hospital.

\section{Compliance with ethical standards}

Conflict of interest The authors declare that they have no conflict of interest.

Publisher's note: Springer Nature remains neutral with regard to jurisdictional claims in published maps and institutional affiliations.

\section{References}

1. Vongphanit J, Mitchell P, Wang JJ. Prevalence and progression of myopic retinopathy in an older population. Ophthalmology. 2002;109:704-11.

2. Liu HH, Xu L, Wang YX, Wang S, You QS, Jonas JB. Prevalence and progression of myopic retinopathy in Chinese adults: the Beijing Eye Study. Ophthalmology. 2010;117:1763-8.

3. Katz J, Tielsch JM, Sommer A. Prevalence and risk factors for refractive errors in an adult inner city population. Invest Ophthalmol Vis Sci. 1997;38:334-40.

4. Gao LQ, Liu W, Liang YB, Zhang F, Wang JJ, Peng Y, et al. Prevalence and characteristics of myopic retinopathy in a rural Chinese adult population: the Handan Eye Study. Arch Ophthalmol. 2011;129:1199-204.

5. Rada JA, Shelton S, Norton TT. The sclera and myopia. Exp Eye Res. 2006;82:185-200. 
6. Harper AR, Summers JA. The dynamic sclera: extracellular matrix remodeling in normal ocular growth and myopia development. Exp Eye Res. 2015;133:100-11.

7. McBrien NA, Gentle A. Role of the sclera in the development and pathological complications of myopia. Prog Retin Eye Res. 2003;22:307-38.

8. McBrien NA, Jobling AI, Gentle A. Biomechanics of the sclera in myopia: extracellular and cellular factors. Optom Vis Sci. 2009;86:E23-30.

9. VanderBeek BL, Johnson MW. The diversity of traction mechanisms in myopic traction maculopathy. Am J Ophthalmol. 2012;153:93-102.

10. Gaucher D, Haouchine B, Tadayoni R, Massin P, Erginay A, Benhamou N, et al. Long-term follow-up of high myopic foveoschisis: natural course and surgical outcome. Am J Ophthalmol. 2007;143:455-62.

11. Ikuno Y, Sayanagi K, Soga K, Oshima Y, Ohji M, Tano Y. Foveal anatomical status and surgical results in vitrectomy for myopic foveoschisis. Jpn J Ophthalmol. 2008;52:269-76.

12. Fang X, Weng Y, Xu S, Chen Z, Liu J, Chen B, et al. Optical coherence tomographic characteristics and surgical outcome of eyes with myopic foveoschisis. Eye. 2009;23:1336-42.

13. Ikuno Y, Sayanagi K, Ohji M, Kamei M, Gomi F, Harino S, et al. Vitrectomy and internal limiting membrane peeling for myopic foveoschisis. Am J Ophthalmol. 2004;137:719-24.

14. Kumagai K, Furukawa M, Ogino N, Larson E. Factors correlated with postoperative visual acuity after vitrectomy and internal limiting membrane peeling for myopic foveoschisis. Retina. 2010;30:874-80.
15. Zhang Z, Wei Y, Jiang X, Zhang S. Pars plana vitrectomy and wide internal limiting membrane peeling with perfluoropropane tamponade for highly myopic foveoschisis-associated macular hole. Retina. 2017;37:274-82.

16. Kwok AK, Lai TY, Yip WW. Vitrectomy and gas tamponade without internal limiting membrane peeling for myopic foveoschisis. Br J Ophthalmol. 2005;89:1180-3.

17. Shimada N, Tanaka Y, Tokoro T, Ohno-Matsui K. Natural course of myopic traction maculopathy and factors associated with progression or resolution. Am J Ophthalmol. 2013;156:948-57 e1.

18. Lai TT, Ho TC, Yang CM. Spontaneous resolution of foveal detachment in traction maculopathy in high myopia unrelated to posterior vitreous detachment. BMC Ophthalmol. 2016;16:18.

19. Hirakata A, Hida T. Vitrectomy for myopic posterior retinoschisis or foveal detachment. Jpn J Ophthalmol. 2006;50:53-61.

20. $\mathrm{Wu} \mathrm{TY}$, Yang $\mathrm{CH}$, Yang $\mathrm{CM}$. Gas tamponade for myopic foveoschisis with foveal detachment. Graefe's Arch Clin Exp Ophthalmol. 2013;251:1319-24.

21. Ng DS, Cheung CY, Luk FO, Mohamed S, Brelen ME, Yam JC, et al. Advances of optical coherence tomography in myopia and pathologic myopia. Eye. 2016;30:901-16.

22. Goeminne P, Salvetti P, Cardoen L, Hamon F. Improved detection of unexpected epiretinal membranes (ERM) in high myopia with scanning laser opthalmoscopy Heidelberg (SLO) and optical coherence tomography (OCT3). Invest Ophthalmol Vis Sci. 2004;45:2982.

23. Soreide K, Korner H, Soreide JA. Diagnostic accuracy and receiver-operating characteristics curve analysis in surgical research and decision making. Ann Surg. 2011;253:27-34. 